\title{
COVID-19 and diabetes: What have we learned so far?
}

\author{
Authors: Nida Taher, ${ }^{A}$ Mohammed SB Huda ${ }^{B}$ and Tahseen A Chowdhury ${ }^{B}$
}

\begin{abstract}
COVID-19 and diabetes are both pandemics with major impacts on global public health. While the response to COVID-19 has been rapid and progressive to reduce risk of harm, the response to the diabetes pandemic has been somewhat more muted. People with diabetes have been disproportionately affected by COVID-19, with growing evidence of higher mortality and morbidity. In this article, we discuss the impact of COVID-19 on our diabetes service in an urban area in the UK. We discuss the impact on our patients and ourselves, and the possible lessons we can carry into the future.
\end{abstract}

KEYWORDS: Diabetes, COVID-19

DOI: $10.7861 /$ clinmed.2020-0261

\section{Introduction}

The two pandemics of coronavirus disease 2019 (COVID-19) and diabetes have sharply contrasting features. COVID-19, caused by the severe acute respiratory syndrome coronavirus-2 (SARSCoV-2), is a newly emerged disease that has become a global public health emergency. The outbreak of COVID-19 has led to the implementation of extraordinary public health measures throughout the world in a short space of time. At the time of writing, the condition has caused more than 300,000 deaths. ${ }^{2}$

By contrast, however, diabetes is a slowly evolving pandemic and is now a leading cause of mortality and morbidity, causing 4.2 million deaths worldwide in 2019. ${ }^{3}$ Currently there are 425 million people living with the condition, $90 \%$ of whom have type 2 diabetes (T2D), and an estimated 352 million with high risk of diabetes from impaired glucose regulation. ${ }^{3}$ By 2045 the number of people with diabetes is expected to have risen by almost $50 \%$ worldwide. A person dies every 7 seconds from diabetes or its related complications, with over half of those deaths occurring under the age of 60 years.

As diabetes is common, many people living with the condition will have developed COVID-19. In this article, we discuss the impact of the SARS-CoV-2 epidemic on patients with diabetes and a diabetes care system in the UK. We aim to review what we have learned so far in a large urban hospital in London, UK.

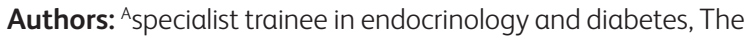
Royal London Hospital, London, UK; ${ }^{B}$ consultant in diabetes, The Royal London Hospital, London, UK

\section{Diabetes led to poorer outcomes in patients with COVID-19}

As the pandemic grew, a number of risk factors for severity of disease emerged. Of these, the presence of diabetes mellitus (predominantly T2D) and hypertension emerged as among the strongest risk factors for severity of SARS-CoV-2 infection. ${ }^{4}$ In a study of intensive care patients with COVID-19 in China, 22\% of 32 patients who died had diabetes as a comorbidity. ${ }^{5}$ Risk of admission to intensive care, as a surrogate for severity of disease, doubled in patients with diabetes. ${ }^{6}$ Further studies have suggested that $12-16 \%$ of patients with severe disease had diabetes, 7,8 and mortality was around three-fold higher in people with diabetes compared to non-diabetic individuals. ${ }^{9,10}$ Indeed, patients with multiple comorbidities including diabetes, hypertension and chronic respiratory diseases had poorer outcomes. ${ }^{9,10}$ Recent data suggest that admission hyperglycaemia predicted severity of radiological findings in patients with COVID-19.11 This mirrors previous data on outbreaks of coronavirus infections (SARS and Middle East Respiratory Syndrome (MERS)), and severe influenza A H1N1 pandemics, which also showed that diabetes was an important risk marker for adverse outcomes. . $^{12-14}$

It is unclear why diabetes should offer such a potent increase in risk for COVID-19 and other viral infections. Diabetes does appear to be a state of impaired immune responsiveness, and poor glucose control appears to impair several aspects of the immune response to viral infection, while also increasing the risk of secondary bacterial infection. ${ }^{15,16}$ Interestingly, there is little evidence that people with type 1 diabetes (T1D) are affected to the same extent, which suggests that factors independent of glucose are important in increasing risk.

An additional factor may be central obesity (frequently preceding or accompanying the diagnosis of $T 2 D$ ), which increases the risk of adverse outcomes, and this is independent of diabetes status. ${ }^{17}$ It is hypothesised that this may be due to chronic low grade inflammation accompanying obesity, along with release of adipocytokines such as tumour-necrosis factor-alpha or transforming growth factor-beta, which may impair immune response. ${ }^{18}$ Obesity-associated comorbidities such as obstructive sleep apnoea or heart failure may also impair respiratory capacity, thereby increasing difficulties with ventilation. ${ }^{19}$

In addition, diabetes-related complications such as chronic kidney disease (CKD) or ischaemic heart disease (IHD) may have contributed to poorer outcomes. Indeed, acute kidney injury (AKI) has been established as an important adverse factor in COVID-19 outcomes, ${ }^{20}$ and patients with diabetes have a higher risk of AKI. ${ }^{21} \mathrm{~A}$ further area of interest has been the use of angiotensin 
converting enzyme inhibitors (ACEIs) and angiotensin receptor blockers (ARBs), which are widely used in people with diabetes as they have proven cardio- and reno-protective effects. ACEI or ARB therapy leads to increased expression of ACE-2, and it appears that SARS-CoV-2 spike protein binds to cells through ACE- $2,{ }^{22}$ and hence prior exposure to these drugs may hypothetically increase risk of infection. There is, as yet, not enough evidence to advocate cessation of these therapies in people at high risk of SARS-CoV-2. ${ }^{23}$

There is now growing evidence that SARS-CoV-2 infections disproportionately affect people of black, Asian and minority ethnic (BAME) origin. ${ }^{24}$ There may be many reasons for this, but one factor may be the increased risk of cardio-metabolic diseases, including T2D, in this population.

\section{Diabetic emergencies were common in patients with COVID and needed imaginative treatment}

The human endocrine pancreas widely expresses ACE-2, and it is hypothesised that the virus may infect islet cells and cause acute $\beta$-cell dysfunction, leading to acute hyperglycaemia. ${ }^{25}$ The SARS virus showed some direct toxic effect on pancreatic islets, reducing insulin releasing capacity, ${ }^{26}$ and a similar phenomenon may occur with SARS-CoV-2.

There is emerging evidence of increased risk of acute hyperglycaemic emergencies as a presentation of COVID-19. In a study of 658 hospitalised patients with confirmed COVID-19, 42 $(6.4 \%)$ presented with ketosis, of whom 15 had diabetes, and three developed diabetic ketoacidosis (DKA). ${ }^{27}$ Development of ketosis was associated with adverse outcomes in patients with or without diabetes. Case reports from South Korea of DKA and hyperosmolar hyperglycaemic syndrome (HHS) in patients with COVID-19 have been published. ${ }^{28}$

At the height of the epidemic affecting our hospital in East London, we noted a significant rise in cases of hyperglycaemic emergencies associated with proven or strongly suspected COVID-19 infection. Our rate of diabetic emergencies rose seven-fold during this period, compared to the period 2013-2018 (unpublished data). These cases are awaiting further analysis, but of note is the fact that many cases of HHS were associated with marked ketosis, and some cases of DKA were seen in patients with previously well controlled T2D.

At the time, management of these conditions needed rapidly evolving paradigms as experience grew. As a result, clinicians managing such cases collaborated in developing new guidelines on their management, and these were disseminated widely to enable areas of the country less affected by the pandemic to adjust their treatment guidelines. ${ }^{29}$ One concern was that of fluid overload in such patients, which might have adverse impacts on their ventilatory capacity, and hence fluid regimens were slowed down in certain circumstances. As COVID-19 appeared to be a condition increasing risk of coagulopathy, consideration was given to early anticoagulation to reduce the risk of thromboembolism. Imaginative use of subcutaneous insulin regimens was considered due to the lack of infusion pumps available for intravenous insulin therapy at the height of the crisis. In addition, as sodium-glucose transporter-2 inhibitor (SGLT-2i) drugs are associated with an increased risk of DKA, it was suggested that these drugs (as well as metformin) should be stopped immediately on admission in any patient with diabetes presenting with COVID-19 symptoms. ${ }^{30}$

\section{Diabetes clinicians were able to work outside their comfort zone}

In the UK, most consultants and trainees in endocrinology and diabetes are dual-accredited in general (internal) medicine. Therefore, diabetes clinicians have been at the forefront of the SARS-CoV-2 crisis, and have very readily adapted to fulfil the needs of the NHS during this crisis. In our own unit, all non-emergency clinical activity was cancelled for a period of two months, meaning that all routine diabetes clinics were cancelled or converted to telephone/video consultations.

Rotas changed such that consultants and trainees were fully embedded with acute and emergency care, often working punishing shifts. As generalists, we have been used to managing complex, multi-morbid patients with general medical problems. Our expertise in the management of patients with COVID-19 grew rapidly as the crisis spiralled. In our unit, diabetes specialist nurses and other members of the multidisciplinary team were seconded to acute areas according to workforce needs. All face-to-face diabetes clinics were cancelled, but clinic lists were reviewed by consultants using a 'red-amber-green' triage of patients who were deemed suitable for urgent, intermediate or routine review, with urgent patients being contacted by telephone or video to ensure their safety. We have continued to run an emergency diabetes service, with telephone and video consultation, and occasional urgent face-to-face consultations as required. A Monday to Friday daily advice line for our patients with diabetes was provided, and well used by our patients. A diabetes antenatal clinic has needed to continue, with a reduced but significant number of face-to-face consultations. Running postnatal oral glucose tolerance tests (OGTT) has been particularly challenging, and we have found mitigating measures such as running OGTT clinics on several sites and reducing numbers to 8-10 to allow social distancing in the waiting area. A well-established community diabetes facilitation has also been suspended, but consultants have continued to offer advice via email, advice and guidance services and telephone. Meetings by Zoom or MS Teams have also been an effective and efficient way to undertake meetings with the wider multidisciplinary team.

Teaching and training of junior doctors has suffered, but in our unit we have taken opportunities to undertake 'micro-teaching' of small groups rotating on our wards, which has been much appreciated by junior doctors at the forefront of care delivery.

Our area has a high number of Muslim patients with T2D, many of whom have wished to fast for Ramadan. Our yearly Ramadanfocused education has had to be curtailed, but our nurses and educator staff have been proactive in contacting and educating patients by telephone to reduce their anxiety at this troubling time. We have also advised them on the care they should take if developing COVID-19 symptoms during this period. ${ }^{31}$

\section{The legacy of diabetes clinic delivery}

Perhaps the enduring legacy of the SAR-CoV-2 pandemic to our service is the establishment of alternative methods of service delivery, including video and telephone consultations. While telephone consultations have been the mainstay of diabetes nurse specialists for many years, many consultants and diabetes trainees have been slow to adopt these methods of consultation in our area. This is likely to change. We recognise the benefit and convenience to patients of a more patient-centred approach, using 
remote working. Management of diabetes, particularly T1D, is undergoing a major change in response to emerging technologies for insulin delivery and glucose monitoring. ${ }^{32}$ Many of these technologies can be shared between clinicians and patients in online platforms, making video/telephone consultations feasible and convenient for clinicians and patients alike.

Our patients have appreciated a friendly phone call from a doctor concerned about their care. There have been opportunities for support, signposting where additional help can be accessed, and offering good wishes for the future. We have been immensely moved by expressions of kindness and support from our patients a frequent refrain of 'keep safe, doctor' has reminded us exactly why we do this job. Once the clinical service is re-opened, it will be interesting to see whether patients will come back to hospitals until they are sure that further COVID-19 cases are not resurfacing.

A further legacy perhaps to reflect on is the fact that our patients with poor glucose control, obesity and other comorbidities have been significantly affected. COVID-19 has hit the headlines as a rapidly overwhelming pandemic needing urgent action. The pandemic of diabetes perhaps is less on the radar as it is slower in onset, but in fact it is far more virulent, as our introductory paragraph illustrates. Nevertheless, the diabetes pandemic needs urgent and concerted action, particularly amongst high risk groups such as those from BAME groups and the socially disadvantaged. It is surely time to develop a concerted public health policy to deal effectively with the obesity and diabetes epidemic affecting so many areas of the country.

\section{Conclusions}

COVID-19 took us all by surprise. Few of us could have imagined the impact of this pandemic on the NHS and wider society. Our patients with diabetes, particularly those of BAME origin, appear to have taken the greatest burden of the disease. Many patients and their families have suffered terribly.

But some positives may come from the episode. We have been surprised at the agility of management and the ability of services to adapt to the crisis. The 'can do' approach, as opposed to the stiflingly bureaucratic and top-down approach so frequently seen in the NHS, has been refreshing, and we really hope that this approach persists in the future. Rapid and facilitated discharge of well patients awaiting social care has been a huge success. Working together in team, re-igniting a team spirit that has been so lacking in recent years, has been a huge positive. We hope that flexibility of approach in consultation and in team meetings is here to stay.

An important lesson to take forward when the dust settles is to consider seriously how we can reduce diabetes risk by public health measures to improve population health via healthy eating and increased physical exercise.

While we are not out of the woods, concerning the NHS response to the crisis, there is much to be heartened about. As a service, we feel we have learnt much about our patients, ourselves and our teams, and hope we can take these lessons forward positively for the future.

\section{References}

1 Munster V], Koopmans M, van Doremalen N, van Riel D, de Wit E. A novel coronavirus emerging in China - key questions for impact assessment. N Engl J Med 2020;382:692-4.
2 Worldometer. COVID-19 coronavirus pandemic. www.worldometers.info/coronavirus/ [Accessed 4 May 2020].

3 International Diabetes Federation. IDF Diabetes Atlas, 8th Edition. www.idf.org/e-library/epidemiology-research/diabetes-atlas/134-idf-diabetes-atlas-8th-edition.html [Accessed 4 May 2020].

$4 \mathrm{Hu}$ Y, Sun J, Dai Z et al. Prevalence and severity of corona virus disease 2019 (COVID-19): A systematic review and meta-analysis. J Clin Virol 2020;127:104371.

5 Yang X, Yu Y, Xu J et al. Clinical course and outcomes of critically ill patients with SARS-CoV-2 pneumonia in Wuhan, China: a single-centered, retrospective, observational study. Lancet Respir Med 2020;8:475-81.

6 Li B, Yang J, Zhao F et al. Prevalence and impact of cardiovascular metabolic diseases on COVID-19 in China. Clin Res Cardiol 2020:109:531-8.

7 Guan W], Ni ZY, Hu Y et al. Clinical characteristics of coronavirus disease 2019 in China. N Engl J Med 2020;382:1708-20.

8 Zhang J], Dong X, Cao YY et al. Clinical characteristics of 140 patients infected with SARS-CoV-2 in Wuhan, China. Allergy 2020, in press (doi: 10.1111/all.14238).

9 Yang J, Zheng Y, Gou X et al. Prevalence of comorbidities in the novel Wuhan coronavirus (COVID-19) infection: a systematic review and meta-analysis. Int J Infect Dis 2020;94:91-5.

10 Zheng Z, Peng F, Xu B et al. Risk factors of critical \& mortal COVID-19 cases: A systematic literature review and meta-analysis. J Infect 2020;pii:S0163-4453(20)30234-6.

11 Iacobellis G, Penaherrera CA, Bermudez LE, Bernal Mizrachi E. Admission hyperglycemia and radiological findings of SARS-CoV-2 in patients with and without diabetes. Diabetes Res Clin Pract 2020;164:108185.

12 Yang JK, Feng Y, Yuan MY et al. Plasma glucose levels and diabetes are independent predictors for mortality and morbidity in patients with SARS. Diabet Med 2006;23:623-8.

13 Wang W, Chen H, Li Q et al. Fasting plasma glucose is an independent predictor for severity of H1N1 pneumonia. BMC Infect Dis 2011;11:104.

14 Memish ZA, Perlman S, Van Kerkhove MD, Zumla A. Middle East respiratory syndrome. Lancet 2020;395:1063-77.

15 Geerlings SE, Hoepelman AI. Immune dysfunction in patients with diabetes mellitus (DM). FEMS Immunol Med Microbiol 1999;26: 256-65.

16 Critchley JA, Carey IM, Harris T et al. Glycemic control and risk of infections among people with type 1 or type 2 diabetes in a large primary care cohort study. Diabetes Care 2018:41:2127-35.

17 Kalligeros M, Shehadeh F, Mylona EK et al. Association of obesity with disease severity among patients with COVID-19. Obesity (Silver Spring) 2020, in press (doi: 10.1002/oby.22859).

18 Almond MH, Edwards MR, Barclay WS, Johnston SL. Obesity and susceptibility to severe outcomes following respiratory viral infection. Thorax 2013;68:684-6.

19 Dixon AE, Peters $U$. The effect of obesity on lung function. Expert Rev Respir Med 2018;12:755-67.

20 Pei G, Zhang Z, Peng J et al. Renal involvement and early prognosis in patients with COVID-19 pneumonia. Am Soc Nephrol 2020;pii:ASN.2020030276.

21 Hodgson LE, Sarnowski A, Roderick PJ et al. Systematic review of prognostic prediction models for acute kidney injury (AKI) in general hospital populations. BMJ Open 2017;7:e016591.

22 Hoffmann M, Kleine-Weber H, Schroeder S. SARS-CoV-2 cell entry depends on ACE2 and TMPRSS2 and is blocked by a clinically proven protease inhibitor. Cell 2020; 181:271-80.

23 Sriram K, Insel PA. Risks of ACE inhibitor and ARB usage in COVID-19: evaluating the evidence. Clin Pharmacol Ther 2020, in press (doi:10.1002/cpt.1863.)

24 Khunti K, Singh AK, Pareek M, Hanif W. Is ethnicity linked to incidence or outcomes of COVID-19? BMJ 2020;369:m1548. 
25 Bindom SM, Lazartigues E. The sweeter side of ACE2: physiological evidence for a role in diabetes. Mol Cell Endocrinol 2009;302: 193-202.

26 Yang JK, Lin SS, Ji XJ, Guo LM. Binding of SARS coronavirus to its receptor damages islets and causes acute diabetes. Acta Diabetol 2010;47:193-9.

27 Li J, Wang X, Chen ] et al. COVID-19 infection may cause ketosis and ketoacidosis. Diabetes Obes Metab 2020, in press (doi: 10.1111/dom.14057).

$28 \operatorname{Kim}$ NY, Ha E, Moon JS, Lee YH, Choi EY. Acute hyperglycemic crises with coronavirus disease-19: case reports. Diabetes Metab ] 2020:44:349-53.

29 National Inpatient Diabetes COVID-19 Response Group. Guideline for managing DKA using subcutaneous insulin (where intravenous insulin infusion is not possible). https://abcd.care/resource/ concise-advice-inpatient-diabetes-during-covid-19-guidelines-mana ging-dka-using [Accessed 4 May 2020].
30 National Inpatient Diabetes COVID-19 Response Group. Front doo guidance. Available from https://abcd.care/resource/concise-adviceinpatient-diabetes-during-covid-19-front-door-guidance [Accessed 4 May 2020].

31 Hanif S, Ali SN, Hassanein M, Khunti K, Hanif W. Managing people with diabetes fasting for Ramadan during the COVID-19 pandemic: a South Asian Health Foundation update. Diabet Med 2020, in press (doi: 10.1111/dme.14312).

32 McAdams BH, Rizvi AA. An overview of insulin pumps and glucose sensors for the generalist. J Clin Med 2016;5:5.

Address for correspondence: Prof Tahseen A Chowdhury, 7th Floor, John Harrison House, The Royal London Hospital, Whitechapel, London E1 1BB, UK.

Email: tahseen.chowdhury@nhs.net 